\title{
Spin Effects in High Energy Scattering in a Simple Constituent Model
}

\author{
Homer A. Neal \\ Department of Physics, University of Michigan, Ann Arbor, Michigan 48109-1120 USA \\ Talk Presented at the Coral Gables Conference on Cosmology and \\ Elementary Particle Physics, December 14, 2001
}

\begin{abstract}
In this paper the author presents an overview of the evolution of proton-proton spin physics over the past quarter century and describes recent work on interpreting high energy polarization effects in a constituent scattering model.
\end{abstract}

\section{INTRODUCTORY REMARKS}

It is with considerable trepidation that I take the floor to speak to you today about advances in spin physics because I recall having been asked to address this same subject at the Coral Gables Conference some 24 years ago [1]. Clearly, the audience will have every reason to expect me to address the question "what has happened in the intervening years". Indeed, in keeping with the request of the conference organizers, my presentation today will be more of a retrospective one than a detailed technical review. I will provide references to sources where the original data may be found and I will give a brief overview of some of my ongoing work in studying polarization phenomena in a quark-quark scattering model. My focus will be on reflections related to the evolution of spin physics through the window of proton-proton scattering.

\section{SPIN: ITS HISTORY}

In his reflections, Dirac -- who, parenthetically, quizzed me vigorously about some new data I presented at my talk here in 1977 -- indicated that one of the earliest suggestions that electrons had spin came from Arthur Compton in 1921 [2]. Compton noted that if electrons were not symmetrical many properties of magnetism could be explained. He also noted that tracks in a Wilson Cloud Chamber had unexpected kinks. He reasoned that if the electron had a magnetic moment it would induce a magnetic moment in the surrounding medium and lead to the observed helical motion of the electron.

In the following years there were other indications that new quantum numbers were needed to explain observations. The number of spectral lines observed in atomic spectroscopy seemed to be double what one would expect from standard Bohr-orbit calculations. German physicists labeled the problem the "duplexity problem": 
"Whenever one took an atom and brought in a further electron, the number of states that one got was double what one expected" [2].

In this environment of puzzlement, it was Kronig in early 1925 who first suggested more formally that the electron had an intrinsic spin. The idea was not embraced by Pauli, and Kronig did not pursue the concept further [3]. Additional insights on the Pauli-Kronig exchanges can be found in the Pauli Festschrift volume edited by Fierz and Weisskopf [4].

Later in 1925, however, two Dutch students, Goudsmit and Uhlenbeck, produced a short paper which suggested electrons had spin and gave it to their advisor, Ehrenfest. They then traveled to Haarlem and showed the paper to Lorentz. Lorentz argued that the concept was not sound and could not possibly be correct because of energy considerations. Goudsmit and Uhlenbeck went back to Leiden and asked Ehrenfest to withdraw the paper. But it was too late. Ehrenfest had submitted the paper to Naturwissenshaften [5]. And, as you know, the rest is history.

Surprisingly, a couple of years later, the discovery of the spin of the proton had a similar tentative emergence. David Dennison had submitted a paper on June 3, 1927 on the specific heat of the hydrogen molecule. Two weeks after that submission (June 16,1927 , to be exact), he sent an addendum to the paper in which he pointed out for the first time that "the mixing ratio of $1 / 3: 1$ means that the spin of the proton is $1 / 2$ ...." [3]. Dennison's personal reflections about this work can be found in his memoirs in reference [6].

In the spirit of full disclosure, I must warn the audience that there will be many references in my talk that seem to be directed toward the University of Michigan and its role in the evolution of spin physics. That is no accident, since I am drawing from materials used when I delivered the inaugural talk on the occasion of my being named the Samuel A. Goudsmit Distinguished University Professor at the University of Michigan a couple of years ago. I used that occasion to celebrate the 75 years of excellence of Michigan in spin physics and I want to share examples of those achievements with the audience here today.

Samuel Goudsmit and George Uhlenbeck, the discoverers of electron spin, spent the principal part of their professional careers on the faculty of the University of Michigan. David Dennison, who, as noted above, was the first to publish the finding that the proton had spin of $1 / 2$, spent almost all of his career at the University of Michigan and was, I might add, one of my most revered professors when I did my graduate work in Ann Arbor. Richard Crane, who is one of our distinguished Michigan emeritus faculty, made the first measurement of $\mathrm{g}-2$ for the electron - a measurment that many at the time had labeled as impossible. Oliver Overseth, a University of Michigan emeritus faculty member, made the first alpha measurment for the lambda hyperon. Michael Longo, who is here at the Conference today, and who was my thesis advisor at Michigan, has made a series of important polarization studies, including his recent studies of the cascade hyperon. Finally, another Michigan faculty member who is well known to the Conference attendees is Alan Krisch, who over the decades has led the advances in both polarized targets and polarized beam technologies that have resulted in so many of the new precision data that are now available to the physics community. So, as you can see, celebrating the contributions 
of Michigan researchers to spin physics seems appropriate. However, by no means do I intend to slight today the enormous contributions made by others.

\section{THE EXPERIMENTAL CHALLENGES}

At each level of aggregation, from molecules to atoms to nuclei, there are interesting questions about spin to be explored -- and are being explored. In elementary particle physics we are focused on a more fundamental set of issues. We want to know what happens when two elementary particles collide and what part of the scattering process is due to the spins of the colliding particles. We demand that any rigorous theory of particle interactions also properly explain the role of spin in the interactions. Spin determines the statistics that the interacting particles must obey and we are ever alert to any indication that our understanding of the spin-statistics relationship is not correct. We are also vigilant in insuring that every opportunity is exploited to use spin as a tool in determining the extent to which of our revered conservation laws are obeyed, and these are most directly tested at the level of the elementary particles.

The set of studies I will review here involves the examination of spin effects in a few well defined scattering processes. Namely, what is the role of spin in elastic proton-proton scattering and in inclusive hyperon production.

\section{MEASUREMENT TECHNIQUES}

I will use a set of my earlier experiments to provide examples of the techniques that were available for making spin studies prior to the recent advances in polarized beam and polarized target technologies. Then I will comment on the increased sensitivity made possible by these advances. The ultimate challenge faced by experimenters is, succinctly, how to fix the spins of the initial state particles and measure the spins of the final state particles -- and to know the identities and origins of each particle studied in the reaction. Not every spin experiment has to know all spins in the reaction, but the more spin information you demand, the more insight you gain about the role of spin in the interaction, and the more difficult the experiment becomes.

When I gave my previous talk at the Coral Gables Conference, the premiere tools for fixing the spin of target protons were the lanthanum magnesium nitrate (LMN) and ethylene glycol polarized targets. The operating principle for the LMN target was to polarize magnetic centers in the target and to stimulate via microwave radiation those centers to couple to the "free" protons in the target and flip the proton's spin. A LMN center does this through a joint spin flip, but then it relaxes back to its original spin state in the presence of a large magnetic field and is ready to couple to another "wrong" state proton and flip it. Meanwhile the flipped protons remain in their polarized state for a long period, because of their weak magnetic moment and long relaxation times. The ethylene glycol targets operated on a slightly different principle of so-called thermal mixing.

In our Argonne ZGS intermediate energy polarization study [7] we employed an ethylene glycol target with a maximum target polarization of approximately $40 \%$. This 


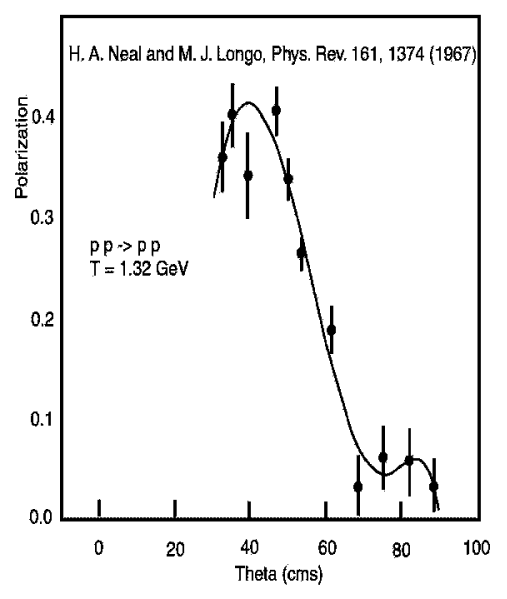

Figure 1: The first observation of the $\mathrm{t}=1(\mathrm{GeV} / \mathrm{c})^{2}$ "double zero" in p-p elastic scattering[11].

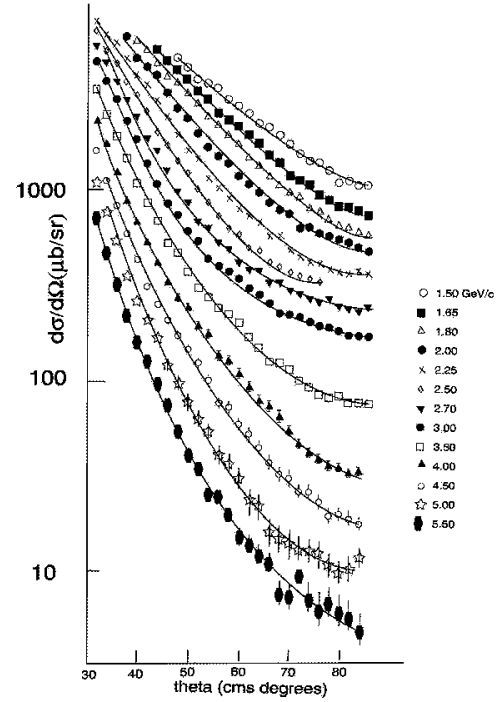

Figure 2: p-p elastic differential cross sections from 1.5 to $5.5 \mathrm{GeV} / \mathrm{c}$. See [1] for data reference.

Michigan staff for more than a decade.

Advances in polarized beams and targets over the decades have been phenomenal -- and this is what has made possible several of the physics results I will review below.

In an experiment at the Brookhaven Cosmotron Mike Longo and I made pioneering measurements of the polarization in $\mathrm{p}-\mathrm{p}$ elastic scattering at energies of approximately $1 \mathrm{GeV}$ using only a carbon analyzer and a polarimeter that tracked its experiment was the first to use a polarized energies. We used a beam of $\sim 5 \times 10^{9}$ protons per pulse, limited to avoid target damage. Moreover, we had to contend with background events because free protons comprised only $\sim 10 \%$ of the target. Today one has targets with polarizations well in excess of $90 \%$ and that are much more radiation resistant. This is a remarkable advance over the time since my last presentation. I must give credit for this to the team of collaborators led by Alan Krisch [8]. proton beams was in its infancy. It was also the determined effort of Alan Krisch and colleagues that made it possible to achieve beams of highly polarized protons at many accelerators (the Argonne ZGS, the Brookhaven AGS, RHIC, and the Indiana Cyclotron). Since there are other talks at this Conference on polarized beams, and since it is a subject well described in the literature [8], I will not go into detail here about the design of these beams. I will mention, however, that one of the great nemeses of polarization beam acceleration is that the beam can become rapidly depolarized by machine imperfections as a given particle passes numerous times through the same imperfection. That problem has been cleverly addressed by two Russian physicists (Derbenev and Kondratenko [9]), who use the machine tune to cause the proton spin to rotate by 180 degrees on each turn around the machine, which causes the effects of the imperfection to be cancelled. referred to as the "Siberian Snake" [10]. By the way, relative to the Michigan involvement in spin physics, Dr. Derbenev has been on the proton target in an external proton beam at high

In 1977 the concept of high energy polarized The technology to accomplish this is commonly 
alignment relative to the incoming beam by using optical spark chambers. An example of the results is shown in Figure 1 [11]. The significance of these measurements is that, for the first time, precision measurements were made beyond 90 degrees cms for $|t|$ values greater than $1(\mathrm{GeV} / \mathrm{c})^{2}$. What we observed was that the polarization assumed a value of zero well before it needed to in satisfying symmetry conditions at 90 degrees $\mathrm{cms}$. We believe this was the first observation of the zero in $p$-p polarizations at $-\mathrm{t}=\sim 1(\mathrm{GeV} / \mathrm{c})^{2}$, a feature which we now know persists all the way into the hundreds of $\mathrm{GeV} / \mathrm{c}$ incident beam momentum. As noted later, I believe this to be due to a transition from the scattering of one quark off one quark to two quarks off two. Unfortunately, we did not know that quarks existed at the time of this measurement. This advance was made simply by using a carbon analyzer, with no polarized targets or beams.

While preparing for additional spin studies early in my years as a faculty member at Indiana University, I noted that the existing cross section data in p-p elastic scattering was still of poor statistical quality. It seemed to make no sense to try to understand polarization effects in an energy region where even the cross section was poorly known, so I temporarily refocused my efforts toward attaining a precise measurement for the p-p cross sections in the intermediate energy regime. At that time the technology of magnetostrictive spark chambers was just being perfected, and we decided to use these chambers for the cross section measurements. The results are shown in Figure 2. One special outcome of these measurements was the discovery of the unusual behavior of the fixed angle cross section, as portrayed in Figure 3. At the

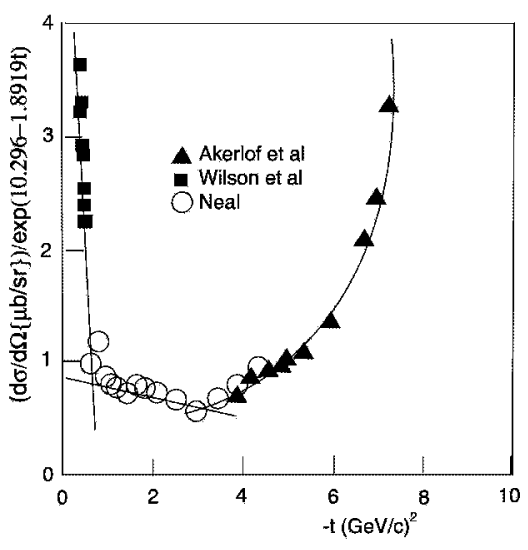

Figure 3: Normalized plot of the differential cross section in p-p elastic scattering at 90 degrees $\mathrm{cm}$. See [1] for data references. time these findings were just regarded as another bizarre rendering of the stubborn and complex p-p system. As discussed later, however, my interpretation now of these breaks is that they too are indicative of the quark structure of the proton.

As previously mentioned, in another experiment I led we used for the first time an ethylene polarized target in an external proton beam to study the polarization in p-p elastic scattering in the $6-12 \mathrm{GeV} / \mathrm{c}$ range. One of our findings from that experiment was a fractal "bump" structure in the polarization [7]. I will refer to this odd feature in a later section as one that gives further credence to our picture of the constituent structure of the proton.

\section{CAN WE SEE QUARK POLARIZATION?}

As I departed for a sabbatical at the Bohr Institute in 1973, in addition to my normal luggage I was burdened by the data described above. The cross section data had shown discontinuities that I did not understand. The polarization data displayed structure which seemed strange and directly correlated with the cross section breaks. As I took up these issues with Holger Nielsen, whom I met early in my stay at the Institute, we 
rather quickly became interested in the possibility that the effects were a manifestation of the quark structure of the proton. Indeed, we had become aware of the work of Landshoff who was having success explaining cross section magnitudes by using an independent quark model [12].

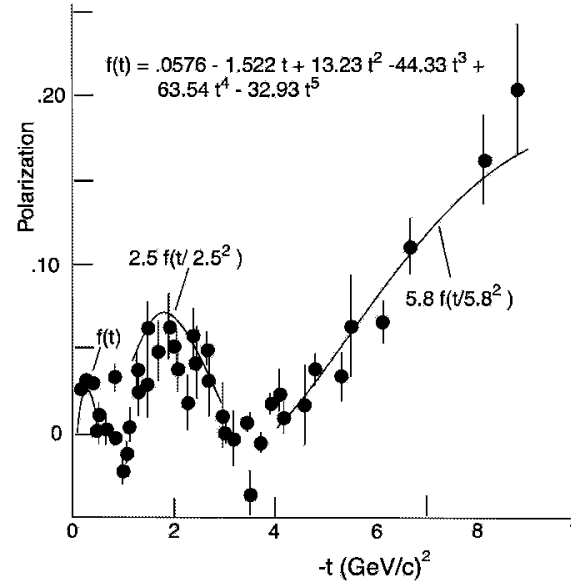

Figure 4: Polarization in p-p elastic scattering at 24 and $28 \mathrm{GeV} / \mathrm{c}$. Reference to data may be found in [14]

The principal postulate we advanced in a Physics Letter article [13] was that within $\mathrm{t}$ ranges where the fixed angle cross section was smooth and continuous in slope, the scattering process was dominated by a fixed number of quarkquark scatters, and as one crossed one smooth region to another the slope change that occurred was due to a change in the number of $\mathrm{q}-\mathrm{q}$ scatters involved (see Figure 3). We also assumed that, to the extent permitted by the quarks combining to make a spin- $1 / 2$ proton, the quarks behaved independently within the proton, they contributed their own polarizations to make up the overall polarization of the proton, and the internal processes that occurred after the quark scattering resulted in the appropriate redistribution of quark momenta but did not affect the overall proton polarization.

This further suggested that in the region we ascribed to a single quark scattering from a single quark $\left(-\mathrm{t}<1(\mathrm{GeV} / \mathrm{c})^{2}\right)$, the proton polarization observed was actually the polarization in single q-q scattering. Armed with the knowledge of what the single $\mathrm{q}-\mathrm{q}$ scattering polarization was, we could then predict what the polarization would be in regions where the dominant scattering mechanism was for 2,3 or more scatters. Indeed, in our 1974 paper we focussed on using the region \#1 data to predict what would happen to the polarization in region \#2 $\left(1<|\mathrm{t}|<3.5(\mathrm{GeV} / \mathrm{c})^{2}\right)$. In that region one would expect the polarization to be roughly $2 \mathrm{P}(\mathrm{t} / 4)$, where $\mathrm{P}(\mathrm{t})$ is the polarization in region \#1. We were impressed with the success of the model in this test. The data beyond region \#2 was so poor at that time that we were content to note that we expected the polarization to be even larger in that region, but we attempted no detailed fits.

As the decades passed and the technological developments for improved polarized targets and beams proceeded, the Krisch group began to accumulate high statistics data in what we call region \#3 ( $\left.-\mathrm{t}>3.5(\mathrm{GeV} / \mathrm{c})^{2}\right)$, the region where we might expect that three quarks would scatter from three quarks [8]. These measurements were the subject of much skepticism in the community in that no mainstream model could explain how spin effects of $>30 \%$ could be present at high energies and large $|t|$. Indeed, most models predicted that in that regime, the polarization should be zero. Our quark-quark scattering model was, to our knowledge, alone in predicting that there should not only be significant spin effects in that region, they should be large, and be simply a scaled version of the polarization in the previous regions. A fit from our 1998 
Physics Letter publication [14] (Figure 4) shows how the model is able to account for the significant structure in the existing p-p polarization data at the energy that, at present, has the largest span of $t$ values in existence. The number of multiple scatters was allowed to vary when the fit shown was generated.

The success of this simple model encourages us to believe that it is the individual quarks inside the proton that are being seen, that there are only three types of scatters (since there seems to be no significant shape changes in the cross section that would signal a fourth region), and that even the spin information in each q-q scattering is preserved and passed on to the scattered proton.

We are often asked if the so-called "spin crisis" [15], which suggests that quarks do not carry all of the proton spin, would invalidate our model. The answer is it does not. The only features of the scattering process that we have invoked is that there are three "clusters" inside the proton that are responsible for the scattering. Each cluster could, for example, be a valence quark plus gluons and the general concept of the model would remain unchanged. The site of the spin is not important.

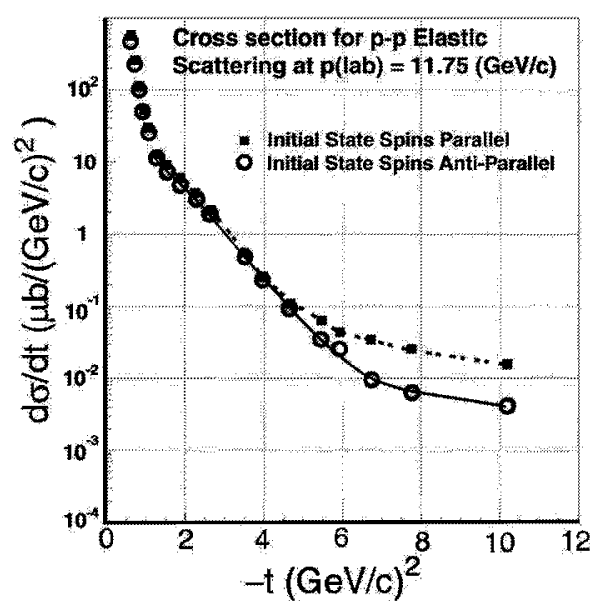

Figure 5: Differential cross section in p-p elastic scattering for the case of the initial state spins being parallel and anti-parallel. Data sources referenced in [16].

\section{ARE SPIN CORRELATION EFFECTS MEASUREABLE AT THE QUARK LEVEL?}

The next challenge we presented the model was to explain the extraordinary behavior observed in the p-p cross section when the two initial protons have their spins parallel vs. anti-parallel. What one notices in the precise $A_{n n}$ data from the Krisch group (Figure 5) is that the two cross sections are comparable up until $|t|$ values are reached that represent the entry into our region \#3. In a paper by Neal and Nielsen published last year in Physics Letters [16], we have argued that, if the basic tenets of the quark interchange model are assumed to be valid, the only way to have three quarks scatter from three quarks and not pay the penalty for spin flips of the protons, is to have the initial state protons have their spins parallel. In such a picture the antiparallel cross section in region \#3 would be expected to just be an extrapolation of the region \#2 cross section. However, with the additional channels available to the parallel cross section, it would be expected to be larger than the anti-parallel values, starting near $-\mathrm{t}=3.5(\mathrm{GeV} / \mathrm{c})^{2}$. So our model even goes some distance in explaining this dramatic effect. 


\section{QUARK POLARIZATIONS AND SPIN EFFECTS IN INCLUSIVE LAMBDA PRODUCTION}

I now would like to say a few words about some current work I am pursuing that seeks to test the applicability of our model to $\Lambda$ inclusive polarizations.

At the time of my last appearance at this Conference it had just been reported that huge polarizations had been observed in $\Lambda$ hyperons which were inclusively produced when $400 \mathrm{GeV} / \mathrm{c}$ protons struck a Be target [17]. This was very puzzling since both the very high energy and the inclusive nature of the production should suggest that spin effects would be small, if they existed at all. To date, this observation, and the many similiar observations for other hyperons, remains a mystery.

I have attempted in recent months to
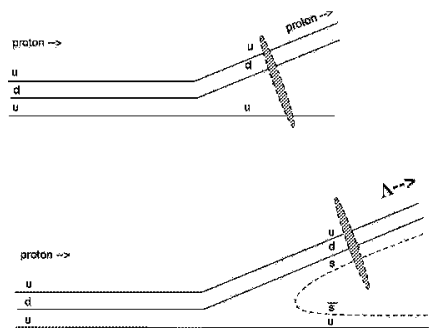

Figure 6: Similarities between p-p elastic scattering and inclusive lambda production.

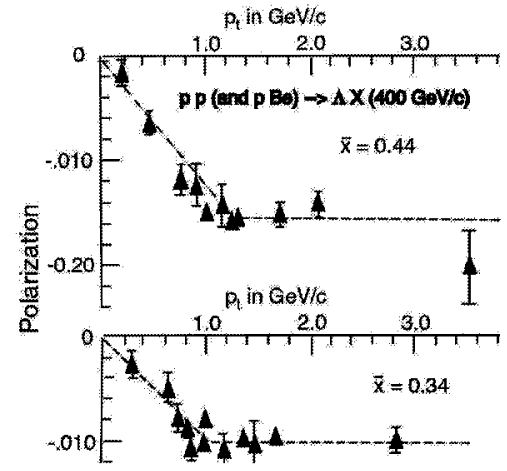

Figure 7: Polarization in lambda inclusive production as function of $p t$ at two different average $x$ values [18] determine just how different the inclusive lambda polarization is from what one would expect based on $p-p$ data. More precisely, since our model purports to be able to extract the $\mathrm{q}-\mathrm{q}$ polarization parameter, one might simply ask the question "at a given $\Lambda \mathrm{p}_{t}$, what would one expect the $\Lambda$ polarization to be if that polarization were due solely to the u,d quarks coming from the incident proton?" Heretofore, this has not been a question that could be simply broached, because mainstream models have no way of asserting directly what the q-q polarization parameter should be. What almost all models have done so far in dealing with the $\Lambda$ polarization matter is to assume that the polarization comes mainly from the s-quark, which is produced from the vacuum as part of a s-sbar pair, as illustrated in Figure 6.

Figure 7 illustrates the remarkable features of the lambda inclusive data, including the prominent features of the large polarization values and a plateau for different values of the Feynman $x$ [18]. Figure 8 shows the measured lambda polarization along with values predicted for the lambda polarization using the $300 \mathrm{GeV} / \mathrm{c}$ p-p polarization from Synder et al in conjunction with our model [19]. Some simple assumptions have been made about how

to scale the momentum of the proton data before overlaying those data on the lambda plot. In essence we require that the $p_{t}$ of the $u, d$ scatters be sufficient to provide the $p_{t}$ needed by the $\mathrm{u}, \mathrm{d}, \mathrm{s}$ and sbar quarks in producing the lambda. The fact that the measured and predicted results agree so well further encourages us in believing the general validity of our model. By allowing some polarization contribution by the squark and allowing it the ability to flip spins with the u,d quarks at low pt, but then 
decreasing that coupling as pt grows -- and noting the requirement for the u,d quarks to ultimately be in a singlet state in the final lambda -- one has the basic ingredients for achieving a flattening in the lambda polarization, also in keeping with observation. This work is ongoing and additional steps are required to rigorously underpin or alter the assumptions advanced above.

\section{ANTICIPATED SPIN STUDIES AT LHC ENERGIES}

Throughout the last few decades, whenever the high energy physics community has faced the prospects of moving to the next higher energy or $|t|$ range there have been many voices suggesting that in the new range spin effects will have died away. Such assertions have always been wrong. As we face the prospects of running at the LHC in a few years, I fully expect that we will again be in an interesting arena where spin effects will be measurable and important in many analyses.

The LHC will produce an enormous number of t-tbar pairs, for example. The relative helicities of these particles will set considerable constraints on the decay distributions of these particles, and will provide sensitive tests of the Standard Model [20].

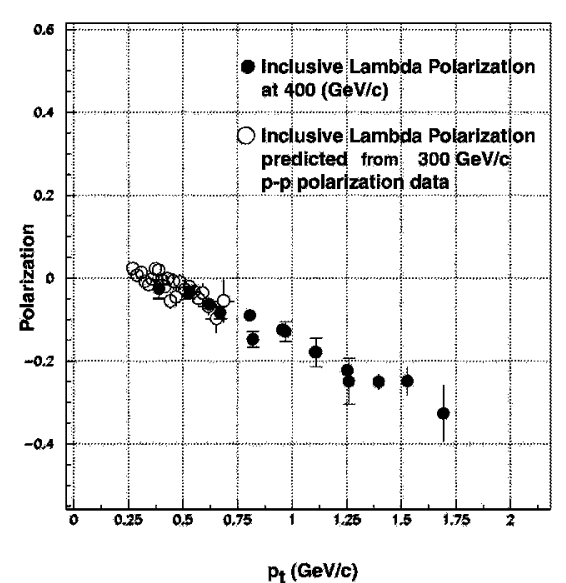

Figure 8: Lambda polarization vs pt for selected $\mathrm{xf}$ ranges, with overlay of predictions derived from p-p data and constituent model (data from Ref [19]).

Also, $\Lambda_{b}$ should be produced in prodigious quantities at the LHC. By using the measured decay properties of $\Lambda_{\mathrm{b}} \rightarrow \psi \Lambda$ and $\psi \rightarrow \mu \mu$, one should be able to determine the polarization of the $\Lambda_{b}$ to $<2 \%$. This may be sufficient to gain some additional insights into how hyperons are inclusively produced, particularly since most analyses suggest that, given the massive nature of the bquark, polarization effects associated with the $\Lambda_{b}$ should be significantly larger than even those of the $\Lambda$ [20].

\section{CONCLUDING REMARKS}

In concluding, I hope I have given you some flavor of the evolution of spin physics, the role it has played in shaping our thoughts about the interactions of particles, and our expectation that it will continue to provide insights as data are acquired at the LHC.

I want to stress that the window I have offered here on spin physics is very narrow. This subfield has so many other components these days, ranging from probes in deep inelastic scattering, e+ e- studies, and polarized structure functions to anticipated new discoveries at RHIC and UNK. Indeed, it is the explosion of the richness of spin studies that may be one of the most notable developments in this area of science over the past quarter-century. 
I want to again salute the members of our community who have advanced the technology in this complex area of our field. I believe that such efforts will eventually allow us to make the measurements necessary to really learn the role of the mysterious spin quantum variable in Nature.

I wish to acknowledge Jens Zorn for his assistance with several of the historical issues referenced in the article. Finally, I wish to acknowledge the conference organizers for sustaining this very special set of scientific meetings over the decades.

\section{REFERENCES:}

1. H. A. Neal, Advances in the Study of Spin Effects in Nucleon-Nucleon Scattering at Small and Intermediate Momentum Transfers in New Frontiers in High Energy Physics, Edited by Behram Kursunoglu, Arnold Perlmutter, and Linda F. Scott (Plenum Publishing Corporation, 1978).

2. Dirac, P. 1975. In Proc. Summer Study High Energy Physics. Polarized Beams, ed. J. Roberts, Argonne National Lab. Rep. HEP-75-02.

3. 3)The Story of Spin, Sin-Itiro Tomonaga, University of Chicago Press, 1997, pp. 258.

4. 4) "Theoretical Physics in the $20^{\text {th }}$ Century, A Memorial Volume to Wolfgang Pauli", Edited by M. Fierz and V.F. Weisskopf. New York, Interscience (1960) 328p

5. Goudsmit, S. , Uhlenbeck, G. 1925. Naturwissenschaften 13:953.

6. D.M. Dennison, "Recollections of Physics and of Physicists During the 1920s", Am. J. Phys. 42, 1051-1056 (1974).

7. Abshire, G.W. et al, Physical Review D 9 (1974) 555.

8. R.C. Fernow, A.D. Krisch, High Energy Physics with Polarized Beams, Ann. Rev. Nucl. Part. Sci. 1981, 31:107-44.

9. Y. S. Derbenev and A. M. Kondratenko, Part. Accel. 8115 (1978).

10. A. D. Krisch, Physics of Atomic Nuclei, 62, Issue 3, 1999 pp. 479-484.

11. H. A. Neal and M. J. Longo, Phys. Rev. 161, 1374 (1967).

12. P.V. Landshoff, Phys. Rev. D 10 (1974) 1024.

13. H.A. Neal, H.B. Nielsen, Physics Letters B 51 (1974) 79.

14. H.A. Neal, J.B. Kuah, H.B. Nielsen, Physics Letters B 439 (1998) 407. (Note: correct coefficient of $t$ in Fig. 2 in this reference is -1.522 ; correct units for cross section for Figs. 5, 6 are $\mu \mathrm{b} /(\mathrm{GeV} / \mathrm{c})^{2}$.

15. John Ellis, Proceedinsg of the $12^{\text {th }}$ International Conference on High Energy Spin Physics, pg. 7 , 1997, World Scientific.

16. Homer A. Neal and Holger B. Nielsen, Physics Letter B 508 (2001) 251-258.

17. G. Bunce et al, PRL36, 1113 (1976).

18. J. Lach, Hyperon Polarization: An Experimental Overview, Fermilab-Conf-92/378, December, 1992.

19. B. Lundberg et al., Phys. Rev. D40, 3557 (1989); C. Wilkinson et al., Phys. Rev. Let. 46, 803 (1981); J.H. Synder et al, Phys. Rev. Lett. 41, 781 (1978).

20. "ATLAS Detector and Physics Performance Technical Design Report", Vol. II, CERN/LHCC/99-15, ATLAS Collaboration, 25 May, 1999. 